\title{
Inventário Reno de Autoperspectiva RISP: Adaptação Transcultural e Evidência de Validade
}

\author{
Evandro Morais Peixoto ${ }^{1}$ \\ Joel Gagnon ${ }^{2}$ \\ Tami R. Jeffcoat ${ }^{3}$ \\ ${ }^{1}$ Universidade Estadual de Pernambuco, Garanbuns, PE \\ ${ }^{2}$ Universté du Quebéc à Trois Rivieres, Quebec, Canadá \\ ${ }^{3}$ University of Nevada, Reno, Nevada, EUA
}

\begin{abstract}
Resumo
Esta pesquisa teve como objetivos a adaptação transcultural e avaliação das propriedades psicométricas da versão brasileira do Reno Inventory of Self-Perspective - RISP, instrumento que avalia a habilidade de tomada de perspectiva, compreensão de si mesmo enquanto construção contextual, por meio dos fatores enredado, centrado e transcendente. A amostra foi composta por 344 universitários (idade 21,1 \pm 4,8; 64,2\% mulheres). A estrutura interna foi estimada por meio do Exploratory Structural Equation Modeling (ESEM). Também se avaliou a invariância do modelo fatorial entre participantes do sexo masculino e feminino, indicadores de precisão e associação com variáveis externas: satisfação com a vida, fusão cognitiva, ansiedade, estresse e depressão. Os resultados revelaram a estrutura composta por três fatores, conforme hipótese teórica, com indicadores desejáveis de precisão. Foi demonstrado equivalência do modelo de medida ao avaliar participantes dos diferentes sexos, e associações correspondentes as perspectivas teóricas com as variáveis externas estudadas. Os resultados sugerem adequação da versão brasileira RISP.

Palavras-chave: ACT, eu como contexto, flexibilidade psicológica, validade, ESEM
\end{abstract}

Reno Inventory of Self-Perspective RISP: Cross-cultural adaptation and validity evidence

\begin{abstract}
This research aimed to adapt and assess the psychometric properties of the Brazilian version of the Reno Inventory of SelfPerspective (RISP), which aims to measure fusion with self-content, the ability to take a centered self-perspective, and verbal awareness of the transcendent nature of that perspective, using three factors: Entangled, Centered and Transcendent. The sample consisted of 344 Brazilian undergraduate students (age 21.1 $44.8 ; 64.2 \%$ women). The dimensionality of the inventory was estimated by the Exploratory Structural Equation Modeling (ESEM). The invariance of the factorial model between men and women, scale reliability and association with other variables: life satisfaction, cognitive fusion, anxiety, stress and depression were estimated as well. The results showed a three-factor structure, confirming the theoretical hypothesis with desirable precision indices. It was also observed the measurement model equivalence to assess participants of both sexes. The results suggest adequacy of the Brazilian version of the RISP.

Keywords: ACT; Self-as-context; psychologic flexibility; validity, ESEM
\end{abstract}

Inventario Reno de Auto-Perspectiva RISP: adaptación transcultural y evidencias de validez

\begin{abstract}
Resumem
Esta investigación tuvo como principal objetivo la adaptación transcultural y la evaluación de las propiedades psicométricas de la versión brasileña del Reno Inventory of Self-Perspective RISP, un instrumento que evalúa la medición de habilidad de capacidad de toma de perspectiva, comprensión de sí mismo como construcción contextual, a través de los factores Enredado, Centrado y Trascendente. La muestra fue compuesta por 344 universitarios (edad 21,1 \pm 4,8; 64,2\% mujeres). La estructura interna del instrumento fue estimada por la Exploratory Structural Equation Modeling (ESEM). También se evaluó la invariancia del modelo factorial entre participantes de sexo masculino y femenino, indicadores de precisión y asociación con variables externas: satisfacción con la vida, fusión cognitiva, ansiedad, estrés y depresión. Los resultados revelaron la estructura compuesta por tres factores, según hipótesis teórica, con indicadores deseables de precisión. También se demostró la equivalencia del modelo de medida al evaluar participantes de diferente sexo, así como asociaciones correspondientes a perspectivas teóricas con las variables externas estudiadas. Los resultados sugieren adecuación de la versión brasileña RISP.

Palabras clave: ACT; Yo como contexto; flexibilidad psicológica; validez; ESEM
\end{abstract}

A Terapia de Aceitação e Compromisso (Acceptance and Commitement Therapy - ACT) é uma modalidade de terapia cognitivo-comportamental que explica o funcionamento humano por meio da flexibilidade psicológica dos indivíduos. É entendida como a capacidade do sujeito em sentir integralmente o momento atual e manter ou modificar seu comportamento, dependendo dos valores escolhidos por ele (Barbosa \& Murta, 2014; Haeys, Luoma, Bond, Masuda, \& Lillis, 2006; Gillanders et al., 2014). Quando a pessoa vivencia uma situação difícil, seus valores podem ser colocados em segundo plano, promovendo inflexibilidade psicológica. Nela, as 
ações são guiadas pela linguagem, o que pode gerar um comportamento disfuncional (Barbosa \& Murta, 2014). Nesse caso, o indivíduo não consegue agir de maneira flexível no que tange a pensamentos, emoções e sensações avaliadas como negativas.

Silva (2015) aponta que a inflexibilidade psicológica é causa do sofrimento humano e de diferentes tipos de psicopatologias. O objetivo do terapeuta que trabalha com essa abordagem é o de auxiliar o cliente a se tornar mais consciente no que diz respeito aos objetivos de sua própria vida e aos valores que guiam sua conduta, auxiliando-o a lidar com as circunstâncias de mudanças. Nesse sentido, busca-se a flexibilidade psicológica por meio de seis processos centrais na ACT, que são: a atenção presente, valores, compromisso, self como contexto, aceitação e desfusão (Haeys et al., 2006; Hayes, Pistorello \& Biglan, 2008; Gillanders et al., 2014). De acordo com Barbosa e Murta (2014) e Haeys et al. (2006), cada um desses processos possui uma sequência inversa, que promove a inflexibilidade psicológica, como atenção rígida ao passado e/ou ao futuro, objetivos e valores pouco claros, dúvidas e estratégias evasivas, esquiva e fusão cognitiva.

Vandenberghe e Valadão (2013) afirmam que a capacidade de se manter presente no aqui agora pode ser facilitada pela diferenciação entre o "eu-como-conteúdo" que diz respeito ao que o sujeito pensa ou sente em relação a si mesmo, e o "eu-como-perspectiva" que se refere à perspectiva pela qual o sujeito observa seus pensamentos e sentimentos. Nesse sentido, não basta apenas ter um conceito claro a respeito de si, pois há sempre que se lembrar de que isso não passa de pensamentos e que uma alta identificação com eles resultaria em atitudes inflexíveis. Dessa forma, na ACT, o cliente aprende a se afastar de seus pensamentos e sentimentos colocando-se no lugar de espectador, podendo observá-los e, portanto, compreender os seus significados. Para Hayes et al. (2012), as pessoas em contato com o eu-como-contexto se percebem como um lugar onde os pensamentos e sentimentos passam. Nesse sentido, espera-se que o afastamento psicológico propiciado pela observação do próprio ato de observar os conteúdos psicológicos permita enxergar o lugar desses conteúdos na paisagem mais ampla. A pessoa torna-se, portanto, capaz de agir de acordo com suas escolhas e seus valores, e não sob o controle de suas angústias e conflitos (Hayes et al., 2012). Dessa forma, Gird e Zettle (2013) definiram esse construto como a capacidade de testemunhar experiências de vida de uma perspectiva duradoura e estável.
Embora o construto Eu como Contexto seja umas das peças centrais da ACT na busca da flexibilidade psicológica (Hayes, Strosahl, \& Wilson, 2012; Saban, 2015), poucos foram os investimentos para construção de instrumentos de medidas que avaliassem esse construto. Nessa direção, Gird e Zettle (2013) desenvolveram e investigaram evidências de validade e precisão do Quantitative Measure of Self-as-Context, uma escala composta por 11 itens respondidos por meio de escala Likert de 7 pontos. Análise da estrutura interna realizada por meio da análise fatorial exploratória indicou organização dos itens em dois fatores denominados Transcending (transcendente), uma capacidade de acesso a uma perspectiva duradoura a partir da qual a experiência pode ser observada, e Centering (centrado) que diz respeito à capacidade de encontrar estabilidade diante da turbulência emocional.

Por meio da verificação da relação desses fatores com outras variáveis teoricamente associadas a esses construtos, os autores demonstraram evidências de validade com base na relação com outras variáveis externas do tipo convergente, ao estimar índices de correlações positivas moderadas como as variáveis (satisfação com a vida, flexibilidade psicológica e bem-estar, do tipo divergente, ao estimar índices de correlação negativos moderado com medidas de neuroticismo, e do tipo discriminante, ao verificar índices de correlações com magnitudes nulas ou fracas com as variáveis desejabilidade social e religiosidade. Além disso, bons indicadores de precisão foram observados por meio dos índices alfa de Cronbach superiores a 0,8 (Gird \& Zettle, 2013).

Outro instrumento para avaliação do construto Eu como contexto foi idealizado por Jeffcoat (2015), sob a supervisão de Steven C. Hayes na universidade de Nevada, denominado Reno Inventory of Self-Perspective (Inventário Reno de Autoperspectiva) - RISP, o qual tem por objetivo avaliar a habilidade de tomada de perspectiva (do inglês perspective taking). $\mathrm{O}$ instrumento é composto por 13 itens que são estruturados em três diferentes fatores: Entangled (enredado), Centered (centrado) e Trancendent (transcendente). Uma autoperspectiva enredada corresponde a um processo psicológico envolvendo excessiva fusão cognitiva com o conteúdo do pensamento, autorregras, e excessivo envolvimento com eventos emocionais do passado. Uma pessoa com autoperspectiva enredada apresenta dificuldades em diferenciar sua identidade de tais conteúdos e eventos e, portanto, suas respostas atuais são insensíveis às condições apresentadas 
no ambiente. Uma autoperspectiva centrada corresponde a um processo psicológico em que a pessoa pode tomar uma posição experiencial do Eu/Aqui/ Agora, o que resulta em maior habilidade para observar o fluxo de conteúdo no corpo, na mente, nos processos emocionais e no ambiente. Por fim, uma autoperspectiva transcendente é um processo psicológico em que um indivíduo tem a capacidade, não só de ter uma perspectiva de observação centrada, mas também de ter consciência verbal dessa perspectiva como um "eu central" imutável que transcende o conteúdo da experiência: todo o conteúdo cognitivo, incluindo conceitos e avaliações autorreferenciais, lembranças negativas e experiência emocional ou física (exceto a morte) (Jeffcoat, 2015).

Além dos estudos de evidências de validade com base na estrutura interna do RISP, Jeffcoat, Hayes e Szabo (2015) verificaram evidências de validade com base na relação com outras variáveis: flexibilidade psicológica, autoestima, coping, depressão, estresse e ansiedade. Foram observados associações negativas entre o fator Enredado e as variáveis Flexibilidade Psicológica, Autoestima, Coping e Associações Positivas com as variáveis Depressão, Estresse e Ansiedade. O fator Centrado demonstrou comportamento inverso, ou seja, associação positiva com flexibilidade psicológica, autoestima, coping e negativas com depressão, estresse e ansiedade. Por fim, foram observadas associações positivas entre o fator Transcendente e coping. Além disso, observaram a capacidade discriminativa do instrumento quanto à identificação de grupos clínicos diagnosticados com transtornos depressivos ou ansiedade.

Inúmeras pesquisas têm apontado a importância desse construto na compreensão da flexibilidade psicológica e, portanto, na ACT. Contudo, no Brasil ainda se observa uma ausência de instrumentos destinados à avaliação do Eu como contexto, o que impede os pesquisadores da área terem uma compreensão mais abrangente do comportamento dessas variáveis na população brasileira, bem como da maneira como as diferentes formas de autoperspectiva poderiam se relacionar com variáveis indicadoras de saúde mental. A fim de oferecer contribuições para o preenchimento dessas lacunas, esta pesquisa pautou-se nos seguintes objetivos: a) descrever o processo de tradução e adaptação do RISP para o português brasileiro; b) estimar as primeiras evidências de validade com base na estrutura interna, estrutura fatorial, invariância do modelo fatorial em função do sexo dos participantes e precisão do instrumento; c) estimar evidências de validade com base na relação com variáveis externas dos tipos convergente, divergente e discriminante.

\section{Método}

\section{Participantes}

A amostra, por conveniência, foi composta por 344 estudantes universitários, com idades que variavam entre 17 e 47 anos $(M=21,1 \pm 4,8)$, de ambos os sexos (64,2\% mulheres). Quanto ao estado civil, $91,8 \%$ se autodeclararam solteiros; 7,9\% casados; e $0,3 \%$ divorciados. Todos os participantes eram provenientes de diferentes cursos de graduação: 35,2\% bacharelado em Psicologia; 15,4\% licenciatura em Computação; 13,1\% licenciatura em Matemática; 10,7\% licenciatura em Letras; 10,4\% licenciatura em Pedagogia; 9,2\% licenciatura em Ciências Biológicas; e 7,8\% bacharelado em Medicina de uma universidade pública localizada no agreste meridional pernambucano.

\section{Instrumento}

Reno Inventory of Self-Perspective RISP (Jeffcoat, 2015) Inventário composto por 13 itens, respondidos por meio de escala Likert de 7 pontos, com objetivo de avaliar a capacidade de tomada de perspectiva, teoricamente denominada Eu como contexto. Estudos de evidência de validade do instrumento original demonstraram adequação da estrutura interna à proposta teórica, demonstrando organização dos itens em três fatores que representam diferentes tomadas de perspectivas: Enredado, Centrado e Transcendente, todos com bons níveis de precisão, entre 0,87 e 0,89 . Estudo de adaptação da escala para o franco-quebequense demonstrou estabilidade da estrutura fatorial quando aplicado em outra cultura, bem como bons níveis de precisão alfa de Cronbach entre 0,82 e 89 (Gagnon, Martel, Peixoto, Dionne \& Jeffcoat, 2017).

Escala de Depressão Ansiedade e Estresse - DASS 21 (Lovibond \& Lovibond, 1995). Instrumento composto por 21 itens, respondidos por meio de escala Likert de 4 pontos, que objetiva a avaliação de indicadores de depressão, ansiedade e estresse. No Brasil, a DASS-21 foi adaptada e validada para adultos de diversas regiões do país (Machado \& Bandeira, 2013) e para adolescentes (Patias, Machado, Bandeira \& Dell'Aglio, 2016) com resultados que asseguram a estrutura com três fatores e bons indicadores de precisão, entre 0,83 e 0,96 .

Escala de Satisfação com a Vida - ESV (Diener, Emmons, Larsen, \& Griffin, 1985), que visa avaliar 
a satisfação dos respondentes com as suas condições de vida atuais em relação ao padrão de vida estabelecido por eles como desejável. A escala é composta por cinco itens, respondidos em escala do tipo Likert de 7 pontos que variam entre 1 - Discordo plenamente a 7 - Concordo plenamente. No Brasil, estudos referentes à estrutura interna da ESV têm corroborado a proposta original quanto a solução unifatorial e com bons indicadores de precisão, alfa de Cronbach entre 0,72 e 0,80 (Gonçalvez \& Kapczinski, 2008; Gouveia et al., 2003).

Questionário de Fusão Cognitiva - CFQ (Gillanders et. Al., 2014). Tem por objetivo avaliar o grau em que as pessoas tendem a fundir com seus pensamentos. É composto por sete itens respondidos em uma escala Likert de 7 pontos (Nunca verdadeira a Sempre verdadeira). $\mathrm{O}$ respondente assinala a alternativa que melhor representa a maneira como o conteúdo o descreve. Um estudo de validade da versão brasileira, em acordo com a proposta original, verificou disposição dos itens em uma solução unifatorial com bons indicadores de precisão, alfa de Cronbach entre 0,92 e 0,94 (Lucena-Santos, 2017).

Questionário socioeconômico. Possibilitou $\mathrm{O}$ acesso às principais características dos participantes $\mathrm{da}$ pesquisa: idade, sexo, estado civil, curso de graduação.

\section{Procedimentos}

A adaptação do RISP teve início após autorização dos autores da versão original do instrumento. Assim, o instrumento foi traduzido para o português brasileiro por dois pesquisadores bilíngues português-inglês, com familiaridade com a teoria comportamental cognitiva, mais especificamente com a ACT. Um comitê composto por quatro pesquisadores foi desenvolvido para a construção de uma versão síntese das traduções. Devido a objetividade dos itens e o fato de não contarem com expressões que apresentassem alta influência cultural não foram encontradas dificuldades para a construção de uma versão síntese. Em uma etapa subsequente, back-translation, a versão síntese foi traduzida por um profissional para a língua inglesa. De posse dessa versão, especialistas em avaliação psicológica (dois doutores) avaliaram a equivalência entre as versões retraduzidas e versão síntese. Por fim, o instrumento foi apresentado a 12 estudantes universitários para avaliação da clareza e compreensão do instrumento, os quais sugeriram pequenos ajustes, como resumo das instruções para aplicação e exclusão de palavras redundantes, o que levou a melhor compreensão do conteúdo alvo do item, dando origem assim a primeira versão brasileira do RISP. Vale ressaltar que tais procedimentos foram realizados em acordo com as diretrizes internacionais para adaptação transcultural de instrumentos de medida psicológica (AERA, APA, \& NCME, 2014; ITC, 2010).

\section{Considerações Éticas}

A realização da pesquisa, coleta de dados, ocorreu após a aprovação do projeto pelo Comitê de Ética em Pesquisa sob o protocolo (CAAE: 0118.0.272.000-11), bem como após consentimento formal da diretoria da universidade onde os procedimentos foram realizados. Os instrumentos foram administrados de forma coletiva, nas próprias salas de aula, por dois participantes do grupo de pesquisa treinados para a realização das aplicações. Como critério de inclusão na pesquisa considerou-se a necessidade de serem alunos de graduação com idade igual ou superior a 18 anos, bem como o consentimento formal por parte do estudante. Dessa forma, a realização desse procedimento foi antecedida da assinatura do Termo de Consentimento Livre e Esclarecido, e o tempo de aplicações foi de aproximadamente 30 minutos.

\section{Análise de Dados}

Em acordo com objetivos da pesquisa, diferentes procedimentos foram empregados. Para estimação do número de fatores a serem extraídos da versão brasileira do RISP, recorreu-se aos seguintes critérios: Análise Paralela (AP) baseada nos Minimum Rank Factor Analysis (Timmerman \& Lorenzo-Seva, 2011); Método Kaiser-Guttman, número de fatores com eigenvalue superiores a 1; interpretabilidade da solução fatorial e cargas fatoriais superiores a 0,30 (Costello \& Osborne, 2005).

Para avaliação da estrutura interna da escala, recorreu-se ao Exploratory structural equation modeling (ESEM), com método de estimação, Weighted Least Squares Mean- and Variance-adjusted (WLSMV) e rotação oblíqua Geomin. De acordo com Marsh, Morin, Parker e Kaur (2013), esse método agrupa os importantes aspectos da análise fatorial exploratória (AFE) e análise fatorial confirmatória (AFC). Dentre eles, a possibilidade de contar com uma estrutura complexa em que todos os itens podem se correlacionar com todos os fatores, a possibilidade do cálculo de índices de ajustes comumente observados em modelos confirmatórios e avaliação da invariância de modelos fatorais entre grupos (Tomás, Marsh, González-Romá, Valls, \& Nagengast, 2014). Dessa forma, o modelo 
foi testado a partir de índices comumente adotados na literatura: WLSMV $\chi^{2}, d f, \chi^{2} / d f$, RMSEA, CFI e TLI, tendo como parâmetro os seguintes valores $\chi^{2} /$ $d f<3$, RMSEA $<0,06$, CFI e TLI $>0,95$ (Muthén \& Muthén, 2012), essas análises foram realizadas por meio do software Mplus 7.4.

Uma vez estimada a estrutura interna da versão brasileira do RISP, recorreu-se à análise de consistência interna por meio do coeficiente alfa de Cronbach, tendo como referência indicadora de bons níveis de precisão valores superior a 0,7 (Tabachnick \& Fidell, 2012). Por fim, estimou-se evidências de validade convergente, divergente e discriminantes. Para tanto, foram estimados coeficientes de correlação de Pearson com objetivo de verificação da associação entre os diferentes fatores que compõem o RISP e variáveis como satisfação com a vida, fusão cognitiva, depressão, ansiedade e estresse. Como hipótese, esperava-se que o fator Enredado apresentasse relação negativa com satisfação como a vida e relações positivas com fusão cognitiva, depressão, ansiedade e estresse. Por outro lado, esperava-se comportamento inverso do fator Centrado, ou seja, correlações positivas com satisfação coma vida e negativas com fusão cognitiva, depressão, ansiedade e estresse. Por fim, para o fator Transcendente, esperavam-se correlações de baixa magnitude/ausência de correlação com as variáveis estudadas. As análises que foram realizadas com apoio do software estatístico SPSS versão 22.

\section{Resultados}

Antes de submeter o RISP ao ESEM, verificaramse os indicadores de fatorabilidade dos dados disponível por meio da medida de adequação da amostra de Kaiser-Meyer-Olkin (KMO) e teste de esfericidade de Bartlett, os quais apresentaram valores considerados bons $\mathrm{KMO}=0,813$, e $\chi^{2}$ de Bartlett $=(78) 1070,4 p$ $=0,00001$ que, portanto, asseguravam a realização das análises pretendidas.

Por meio dos métodos de estimação do número de fatores a serem extraídos, observou-se a indicação de três fatores quanto à utilização do Kaiser-Guttman, ou seja, número de fatores com eigenvalue superiores a um, e dois fatores quando avaliado por meio de procedimento análise paralela, indicando que apenas dois fatores apresentaram porcentagem de variância explicada superior à variância explicada provenientes das matrizes de correlação estimadas aleatoriamente, 500 matrizes (Timmerman \& Lorenzo-Seva, 2011). Os resultados são apresentados na Tabela 1.

A partir das informações obtidas junto aos métodos de retenção do número de fatores, submete-se os dados ao ESEM, inicialmente forçando a solução em

Tabela 1.

Método de Retenção de Fatores

\begin{tabular}{cccc}
\hline Fatores & Eigenvalue & \% Variância explicada dados reais & $\begin{array}{c}\text { Média das \% variância } \\
\text { explicada dados aleatórios }\end{array}$ \\
\hline $\mathbf{1}$ & $\mathbf{3 , 4 4 3}$ & $\mathbf{3 3 , 9}$ & $\mathbf{1 5 , 7}$ \\
$\mathbf{2}$ & $\mathbf{1 , 6 6 4}$ & $\mathbf{1 4 , 7}$ & $\mathbf{1 4 , 1}$ \\
$\mathbf{3}$ & $\mathbf{1 , 1 1 8}$ & $\mathbf{8 , 8}$ & $\mathbf{1 2 , 7}$ \\
$\mathbf{4}$ & 0,938 & $\mathbf{7 , 9}$ & 11,3 \\
5 & 0,860 & 7 & 10,1 \\
6 & 0,784 & 6,2 & 8,8 \\
7 & 0,745 & 5,3 & 7,6 \\
8 & 0,690 & 5,2 & 6,4 \\
9 & 0,676 & 4,5 & 5,2 \\
10 & 0,597 & 4,2 & 3,9 \\
11 & 0,563 & 1,8 & 2,7 \\
12 & 0,522 & 0,2 & 1,4 \\
13 & 0,397 & 0 & 0 \\
\hline
\end{tabular}


dois fatores, conforme indicado pela AP, basicamente os resultados apresentavam solução que compreendiam um primeiro fator composto pelos itens do fator Enredado, com o acréscimo do item 3, originalmente desenvolvido para compor fator Centrado, e um segundo fator que englobava os itens dos fatores Centrado e Transcendente. Além disso, os índices de ajustes apresentados pelo modelo se classificam como pouco adequados: $\chi^{2}=184,988 ; g l=53 ; \chi^{2} / g l=3,49$; CFI $=$ 0,934; TLI $=0,902$; RMSEA $=0,085($ IC $90 \%=0,072-$ $0,099)$. Posteriormente, foi testada a solução composta por três fatores conforme indicado pelo método Kaiser-Guttman, que apresentou melhor distribuição dos itens, ou seja, maior concordância como perspectiva teórica e, com a proposta original do instrumento, bem como índices de ajustes classificados como muitos bons $\chi^{2}=102,451 ; g l=42 ; \chi^{2} / g l=2,44 ;$ CFI $=0,970 ;$ TLI $=0,944 ;$ RMSEA $=0,065$ (IC 90\% = 0,049-0,081). Os resultados da estrutura são apresentados na Tabela 2 , onde se observa carga fatorial dos itens, indicadores de precisão, correlação entres os fatores e porcentagem de variância explicada.

O primeiro fator foi composto por oito itens, sete deles desenvolvidos originalmente para avaliação do fator Enredado, contudo o item 3, originalmente desenvolvido para o compor o fator Centrado, apresentou carga fatorial igual a 0,601 neste fator. Quanto a variação das cargas fatoriais, observa-se que estas variavam entre 0,534 (item 7) e 0,829 (item 11). O segundo fator a emergir da análise fatorial foi composto por três itens correspondentes, originalmente, ao fator Centrado com cargas fatoriais que variavam entre 0,353 (item 8) e 0,589 (item 5). Por fim, o terceiro fator foi composto por dois itens, originalmente desenvolvidos para avaliação do fator Transcendente, os quais apresentaram cargas fatoriais iguais a 0,437 (item 6) e 0,845 (item 13). Configurando a versão brasileira do RISP.

Tabela 2.

Modelo Fatorial da Versão Brasileira do RISP

\begin{tabular}{cccc}
\hline Itens & Enredado & Centrado & Transcendente \\
\hline 1 & 0,010 & $\mathbf{0 , 5 8 2}$ & 0,000 \\
2 & $\mathbf{0 , 5 5 2}$ & $-0,289$ & 0,062 \\
3 & $\mathbf{0 , 6 0 1}$ & $-0,027$ & $-0,022$ \\
4 & $\mathbf{0 , 7 6 4}$ & 0,108 & $-0,195$ \\
5 & 0,256 & $\mathbf{0 , 5 8 9}$ & 0,003 \\
6 & $-0,010$ & 0,036 & $\mathbf{0 , 4 3 7}$ \\
7 & $\mathbf{0 , 5 3 4}$ & $-0,133$ & $-0,029$ \\
8 & 0,255 & $\mathbf{0 , 3 5 3}$ & 0,029 \\
9 & $\mathbf{0 , 5 8 7}$ & 0,105 & 0,042 \\
10 & $\mathbf{0 , 7 6 5}$ & $-0,269$ & 0,027 \\
11 & $\mathbf{0 , 8 2 9}$ & $-0,002$ & $-0,113$ \\
12 & $\mathbf{0 , 6 1 5}$ & $-0,329$ & $-0,049$ \\
13 & 0,011 & 0,018 & $\mathbf{0 , 8 4 5}$ \\
Corr. Fatores & $\mathrm{F} 1$ & $\mathrm{~F} 2$ & $\mathrm{~F} 3$ \\
F1 & $\mathbf{0 , 9 1 2}$ & & \\
F2 & 0.189 & $\mathbf{0 , 8 7 2}$ & $\mathbf{0 , 8 1 0}$ \\
F3 & 0.177 & 0.335 & 8,60 \\
\% VE & 26,48 & 12,80 & \\
\hline VE total & 47,88 & & \\
\hline
\end{tabular}

Notas. Corr. Fatores = correlação entre fatores; $\%$ VE $=$ Porcentagem de variância explicada por fator; $\%$ VE total = soma da porcentagem da variância explicada. 
Ainda em relação à estrutura interna, verificouse indicadores de invariância do modelo fatorial entre grupos formados em função dos sexos dos participantes, para tanto, foram avaliados os modelos configural (equivalência da estrutura fatorial) e escalar (equivalência nos níveis dos interceptos dos itens). Vale ressaltar, que, por uma limitação do pacote estatístico MPlus, os índices de ajuste não são computados para o modelo métrico no ESEM.

Os resultados obtidos, para o modelo configural: $\chi^{2}=167,321 ; g l=84 ; \chi^{2} / g l=1,99 ;$ CFI $=0,959 ;$ TLI $=0,924$; RMSEA $=0,076($ IC $90 \%=0,059-0,093)$, e modelo escalar: $\chi^{2}=288,853 ; g l=176 ; \chi^{2} / g l=1,64$; CFI $=0,945$; $\mathrm{TLI}=0,951$; RMSEA $=0,061$ (IC 90\% $=0,048-0,073)$, sugerem bons índices de ajustes para ambos os modelos e $\Delta$ entre os índices CFIs inferior a 0,02 , o que indica a equivalência entre os grupos formado por homens e mulheres em relação à estrutura fatorial da escala e os interceptos dos itens.

Os índices de correlação entre os fatores demonstram magnitudes que variam entre ausência de correlação (fatores Enredado e Transcendente), (fator Centrado e Transcendente) e correlação moderada (Enredado e Centrado). Na diagonal da matriz de correlação, também são apresentados os indicadores de precisão, coeficientes alfa de Cronbach dos respectivos fatores, os quais indicam bons níveis de precisão dos fatores que compõem o instrumento, ou seja, todos superiores a 0,8 . Por fim, são apresentadas as porcentagens de variância explicada dos fatores $(26,48 ; 12,80$ e 8,60 , respectivamente) e da escala total 47,88 .

Para estimação das evidências de validade com base na relação com outras variáveis, empregou-se a correlação de Person entre os fatores do RISP e outras variáveis teoricamente associadas: satisfação com a vida, fusão cognitiva, estresse, depressão e ansiedade. Os resultados são apresentados na Tabela 3.

As correlações bivariadas observadas na Tabela 3 indicam associação negativas moderada entre os escores apresentados no fator Enredado e ESV. Assim como correlações positivas fortes com os escores obtidos em fusão cognitiva e depressão, e associações moderadas com as variáveis estresse e ansiedade. Quanto ao fator Centrado, observa-se índices de correlações positivos, significativos e de baixa magnitude, com a variável satisfação com a vida, e negativo com as variáveis fusão cognitiva, estresse, ansiedade e depressão. Por fim, observa-se ausência de correlação entre o fator Transcendente e outras variáveis estudadas: satisfação com a vida, fusão cognitiva, depressão, ansiedade e estresse.

\section{Discussão}

Esta pesquisa teve como principal objetivo a adaptação transcultural do Inventário Reno de Autoperspectiva - RISP para o português brasileiro, bem como a estimação de diferentes evidências de validade do instrumento, para tanto, empregou-se diferentes procedimentos. Tais esforços correspondem ao início do processo de construção de um argumento de validade capaz de sustentar as inferências realizadas a partir dos escores do RISP frente a população brasileira (AERA, APA, \& NCME, 2014).

A partir dos procedimentos estatísticos empregados para retenção de fatores, verificou-se a indicação da estrutura fatorial com dois (AP) e três fatores (KaiserGuttman). O resultado da ESEM obtidos a partir da primeira indicação de retenção de fatores demonstrou que o primeiro grupamento de itens era composto por aqueles originalmente desenvolvidos para avaliação do fator Enredado (2,4,7,9,10,11 e 12) com acréscimo do item 3 (Sinto-me emocionalmente sobrecarregado quando as coisas dão muito errado) inicialmente desenvolvido para compor o fator Centrado (após a inversão/recodificação da pontuação do item). Enquanto o segundo agrupamento era composto pelos itens dos fatores Centrado (itens: 1,5 e 8) e Transcendente (itens: 6 e 13). Tais resultados eram esperados a medidas que a AP, um método robusto de retenção fatores, apresenta uma

Tabela 3.

Indices de Correlação entre os Fatores do RISP e ESV, CFQ e DASS

\begin{tabular}{|c|c|c|c|c|c|}
\hline & ESV & CFQ & Estresse & Ansiedade & Depressão \\
\hline Enredado &,$- 450^{*}$ & ,773* &, $502^{*}$ &, $517^{*}$ &, $632^{*}$ \\
\hline Centrado &, $168^{*}$ &,$- 273^{*}$ &,$- 197^{*}$ &,$- 193^{*}$ &,$- 180^{*}$ \\
\hline Transcendente & ,105 &,- 020 &,- 053 &,- 049 &,- 093 \\
\hline
\end{tabular}

Notas. ${ }^{*}=$ nível de significância de 0,01; ESV = Escala de Satisfação com a Vida; CFQ = Questionário de Fusão Cognitiva. 
fragilidade quanto a tendência subestimação do número de fatores quando estes apresentam correlações moderadas ou altas entre si, assim como em pesquisas como amostras inferiores a 500 participantes (Crawford et al., 2016; Golino \& Epskamp, 2017; Keith, Caemmerer, \& Reynolds, 2016). Além disso, resultados semelhantes foram observados por Jeffcoat (2015), o que levou os pesquisadores a realização de uma segunda fase da pesquisa recorrendo a análise fatorial confirmatória para o teste de melhor ajuste entre as soluções com dois e três fatores, o que culminou na escolha, empiricamente embasada, da solução composta por três fatores.

Nesse sentido, pode-se inferir a adequação na utilização do ESEM, uma vez que esse método proporcionou a avaliação dos índices de ajuste dos modelos estimados, não exigindo, por exemplo, a divisão da amostra para a utilização de métodos exploratórios e confirmatórios, o que levaria a perda do poder dos métodos estatístico ao contar com subamostras e, portanto, com menor representatividade da população (Marsh, 2013; Tomás et al., 2014). Do ponto de vista teórico, também faz sentido a manutenção da estrutura com três fatores, haja vista que o conteúdo dos itens que compõe o fator Transcendente diz respeito, não só a capacidade do indivíduo em tomar uma perspectiva de observação centrada, mas também a capacidade de ter consciência verbal dessa perspectiva como um "eu central" imutável que transcende o conteúdo da experiência, com exceção da morte. Características que explicam, inclusive, a correlação moderada observada com o fator Centrado (Jeffcoat, 2015).

Em relação à configuração da estrutura observada, em especial em relação ao agrupamento do item 3 (Sinto-me emocionalmente sobrecarregado quando as coisas dão errado), no fator Enredado, pesquisadores canadenses encontraram resultados semelhantes ao adaptarem transculturamente o RISP para o francês e estimar as evidências de validade do instrumento frente a população franco-quebequense (Gagnon et al., 2017). O que indica que, em ambas as amostras (brasileira e francoquebequense), o conteúdo do item foi reconhecido de uma tomada de perspectiva, descrição de si mesmo, voltada para o passado, pois, mesmo quando recodificado para se tornar um item positivo, e indicar a capacidade de superação do indivíduo e, portanto, a permanência do foco dele no aqui agora, o item apresentou forte carga fatorial no fator Enredado. Dessa forma, com exceção do item 3, os agrupamentos dos itens obtidos por meio da ESEM com três fatores corresponderam a estrutural fatorial proposta na versão original do instrumento, que compreende três fatores: Enredado, Centrado e Transcendente. Demonstrando equivalência também na porcentagem de variância explicada obtida na versão original do instrumento (49,54\%) (Jeffcoat, 2015), bem como na versão franco-quebequense (53\%).

Quanto aos indicadores de precisão dos fatores que compuseram a versão brasileira do RISP, os índices de consistência interna $(0,912,0,872$ e 0,810 , respectivamente) indicaram valores classificados como bons (Tabachnick \& Fidell, 2012), em relação aos indicadores de precisão do instrumento original esses não puderam ser comparado, haja vista que os autores apresentam somente os índices frente a escala total $(0,89)$. Contudo, os resultados encontrados na versão brasileira do RISP são coerentes com os valores observados por Gagnon et al. (2017) frente à população franco-quebequense $(0,891,0,830$ e 0,820 , respectivamente). O que aponta para as primeiras evidências de precisão da versão brasileira do RISP (AERA, APA, \& NCME, 2014).

Ainda em relação à estrutura interna do instrumento, verificou-se a invariância do modelo de medida, configural e escalar, entre os grupos formados por participantes do sexo feminino e masculino. Os resultados obtidos indicam a capacidade do instrumento em avaliar os diferentes grupos de forma semelhante, quando controlado o nível de traço latente dos participantes, ou seja, que a configuração fatorial e os níveis dos interceptos dos itens são equivalentes entre os grupos (Muthén \& Muthén, 2015). De acordo com literatura especializada, o conhecimento dessa característica da escala é de suma importância aos pesquisadores e profissionais práticos envolvidos com a testagem psicológica, pois são elas que garantem que as variáveis observadas (itens de um teste) se relacionam aos construtos latentes (fatores) de maneira semelhante entre os diferentes grupos, possibilitando assim a comparação entre eles a partir dos escores brutos obtidos junto ao instrumento (Borsboom, 2006; Milfont \& Fisher, 2010).

A última etapa da presente pesquisa consistiu em estimar evidências de validade do RISP baseada na relação com outras variáveis. Os resultados indicam associação negativa entre a o fator Enredado e satisfação com a vida, bem como forte associação positiva com as medidas fusão cognitiva, estresse, ansiedade de depressão. Tais resultados corroboram a perspectivas teóricas que fundamentam o desenvolvimento do RISP, a ACT, haja vista que a tomada de perspectiva voltada para passado levaria o sujeito a uma fusão com os pensamento e sentimento negativos do passado e, portanto, com o Eu como conteúdo, promovendo 
inflexibilidade psicológica, fenômeno que já vem sendo associados ao longo dos anos ao sofrimento psicológicos e psicopatologias (Hayes et al., 2012; Hayes, Strosahl, \& Wilson, 2012; Saban, 2015). Na direção contrária, observa-se associações positivas, de baixa magnitudes, entre o fator Centrado e satisfação com a vida, bem como associações negativas com fusão cognitivas, estresse, ansiedade e depressão. Tais resultados corroboram a hipótese teórica de que a capacidade de tomada de perspectiva centrada, ou seja, a habilidade de experienciar Eu/Aqui/Agora pode contribuir para a flexibilidade psicológica e, portanto, para melhores indicadores de saúde mental (Gillanders et al., 2014; Haeys et al., 2006; Hayes, Pistorello \& Biglan, 2008; Vandenberghe \& Valadão, 2013), por fim, observa-se ausência de associação entre o fator Transcendente e as outras variáveis empregadas neste estudo. O que indica que, embora o sujeito apresente consciência verbal de um "eu central" imutável que transcende o conteúdo da experiência, isso não pode ser visto como mecanismos que garantam o equilíbrio psicológico e, portanto, indicadores de saúde mental. Dessa forma, pode-se inferir que o conjunto de resultados aponta para as primeiras evidências de validade com base na relação com variáveis externas: convergente, divergente e discriminante da versão brasileira do RISP (AERA, APA, \& NCME, 2014).

\section{Considerações Finais}

A presente pesquisa possibilitou a adaptação do RISP para o português brasileiro, bem como a estimação de evidências de validade baseada na estrutura interna e precisão da escala frente a uma amostra de universitários oriundos do agreste pernambucano. Os resultados aqui apresentados apontam para o potencial do instrumento em integrar pesquisas que objetivam a avaliação da tomada de perspectiva da população brasileira, bem como em compor o instrumental de profissionais práticos, em especial, aqueles interessados em desenvolver propostas interventivas com base nos pressupostos da ACT. Por fim, ressalta-se algumas das limitações da pesquisa como o fato de se basear em uma amostra por conveniência oriunda de uma região específica do país, região nordeste. Em pesquisas futuras esforços devem ser realizados no sentido de envolver amostras mais heterogêneas e que abranja outras populações de interesse para além do contexto universitários. Por fim, sugere-se a realização de outros estudos que objetivem, desenvolvimento de normas interpretativas para os possíveis escores advindos da versão brasileira do RISP, bem como estudos transculturais que comparem características das diferentes versões do instrumento (americana, canadense e brasileira).

\section{Referências}

American Educational Research Association (AERA), American Psychological Association (APA), \& National Council on Measurement in Education (NCME). (2014). Standards for educational and psychological testing. Washington, DC: AERA.

Barbosa, L. M., \& Murta, S. G. (2014). Terapia de aceitação e compromisso: história, fundamentos, modelo e evidências. Revista Brasileira de Terapia Comportamental e Cognitiva, 16(3), 34-49.

Borsboom, D. (2006). The attack of the psychometricians. Psychometrika, 71(3), 425-440. doi: 10.1007/ s11336-006-1447-6

Costello, A. B., \& Osborne, J. W. (2005). Best Practices in Exploratory Factor Analysis: Four recommendations for getting the most from your analysis. Practical Assessment, Research, and Evaluation, 10(7), $1-9$

Crawford A. V., Green, S. B., Levy, R., Lo, W. J., Scott. L., Svetina, D., et al. (2010). Evaluation of parallel analysis methods for determining the number of factors. Educational and Psychological Measurement, 70(6), 885-901. doi: 10.1177/0013164410379332

Diener, E., Emmons, R. A., Larsen, R. J., \& Griffin, S. (1985). The Satisfaction with Life Scale. Journal of Personality Assessment, 49(1), 71-75.

Gagnon, J., Martel, M. E., Peixoto, E. M., Dionne, F., \& Jeffcoat, T. (2017). Self-esteem or self-as-context? Validation of the new inventory on self-perspective and comparison with positive sense of self in the explanation of psychological distress in the general population (Manuscript submitted for publication)

Gillanders, D. T., Bolderston, H., Bond, F. W., Dempster, M., Flaxman, P. E., Campbell, L., et al... (2014). The development and initial validation of The Cognitive Fusion Questionnaire. Behavior Therapy, 45(1), 83-101, doi: 10.1016/j.beth.2013.09.001

Gird, S. R., \& Zettle, R. D. (2013). Developing a quantitative measure of self-as-context. GRASP: Graduate Research and Scholarly Projects, 9(1), 47-48. 
Golino, H. \& Epskamp, S. (2017). Exploratory graph analysis: A new approach for estimating the number of dimensions in psychological research. PLoS ONE, 12(6): e0174035. doi: 10.1371/journal. pone. 0174035

Gonçalves, D. M., \& Kapczinski, F. (2008). Prevalência de transtornos mentais em indivíduos de uma unidade de referência para Programa Saúde da Família em Santa Cruz do Sul, Rio Grande do Sul, Brasil. Cadernos de Saúde Pública, 24(9), 2043-2053.

Gouveia, V. V., Chaves, S. S. S., Oliveira, I. C. P., Dias, M. R., Gouveia, R. S. V., \& Andrade, P. R. (2003). A utilização do QSG-12 na população geral: Estudo de sua validade de construto. Psicologia: Teoria e Pesquisa, 19(3), 241-248.

Haeys, S. C., Luoma, J. B., Bond, F. W., Masuda, A., \& Lillis, J. (2006). Acceptance and commitment therapy: Model, processes and outcomes. Behavior Research and Therapy, 44(2006), 1-25, doi: 10.1016/j. brat.2005.06.006

Hayes, S. C., Pistorello, J., \& Biglan, A. (2008). Terapia de aceitação e compromisso: Modelo, dados e extensão para a prevenção do suicídio. Revista Brasileira de Terapia Comportamental e Cognitiva, 10(1), 81-104.

Hayes, S. C., Strosahl, K. D., \& Wilson, K. G. (2012). Acceptance and commitment therapy: The process and practice of mindful change. New York, NY: Guilford.

International Test Commission. (2010). International Test Commission Guidelines for translating and adapting tests. Liverpol: International Test Commission. Recuperado de http://www.intestcom.org

Jeffcoat, T. R. (2015). Development of the Reno Inventory of Self-Perspective (RISP): Measuring Self in the ACT Model (Tese de doutorado não publicada). University of Nevada, Reno.

Jeffcoat, T. R., Hayes, S. C., \& Szabo, T. (2015/ July). Development of the Reno Inventory of SelfPerspective, Measuring Self-as-Context and Perspective Taking. In T. Jeffcoat President (Chair). Continuing Developments in Measuring ACT Processes. Symposium conducted at the meeting of ACBS Annual World Conference 13, Berlin.

Keith T. Z, Caemmerer, J. M., \& Reynolds, M. R. (2016). Comparison of methods for factor extraction for cognitive test-like data: Which overfactor, which underfactor?. Intelligence, 29(54), 37-54.

Lorenzo-Seva, U., Timmerman, M. E., \& Kiers, H. A. L. (2011). The hull method for selecting the number of common factors. Multivariate Behavioral Research, 46, 340-364. doi: 10.1080/00273171.2011.564527

Lovibond, P. F., \& Lovibond, S. H. (1995). The structure of negative emotional states: Comparison of the Depression Anxiety Stress Scales (DASS) with the Beck depression and anxiety inventories. Behaviour Research and Therapy, 33(3), 335-343. doi: 10.1016/j.rbp.2012.05.003

Lucena-Santos, P., Carvalho, S., Pinto-Gouveia, J., Gillanders, D., \& Oliveira, M. S. (2017). Cognitive Fusion Questionnaire: Exploring measurement invariance across three groups of Brazilian women and the role of cognitive fusion as a mediator in the relationship between rumination and depression. Journal of Contextual Behavioral Science, 6(1), 53-62. doi: 10.1016/j.jcbs.2017.02.004.

Lucena-Santos, P., Carvalho, S., Pinto-Gouveia, J., Gillanders, D., \& Oliveira, M. S. (2017). Cognitive Fusion Questionnaire: Exploring measurement invariance across three groups of Brazilian women and the role of cognitive fusion as a mediator in the relationship between rumination and depression. Journal of Contextual Behavioral Science. doi: 10.1016/j.jcbs.2017.02.004

Marsh, H. W., Morin, A. J. S., Parker, P., \& Kaur, G. (2013). Exploratory Structural Equation Modeling: An integration of the best features of Exploratory and Confirmatory Factor Analysis. Annual Review of Clinical Psychology, 10, 85-110. doi: 10.1146/ annurev-clinpsy-032813-153700

Machado, W. L., \& Bandeira, D. R. (2013). Adaptação e validação da Depression, Anxiety and Stress Scale (DASS21) para o Português brasileiro (Manuscrito submetido para publicação).

Milfont, T. L., \& Fisher, R. (2010). Testing measurement invariance across groups: Applications in crosscultural research. International Journal of Psychological Research, 3(1), 111-121. Recuperado de https://dialnet.unirioja.es/descarga/.../3296462.pdf

Muthén, L. K., \& Muthén, B. O. (2012). Mplus User's Guide. Seventh Edition. Los Angeles, CA: Muthén \& Muthén. 
Saban, M. T. (2015). O que é Terapia de Aceitação e Compromisso? Em P. Lucena-Santos, J. Pinto-Gouveia \& M. S. Oliveira (Eds.). Terapias Comportamentais de Terceira Geração (pp.179-216). Sinopsys: Novo Hamburgo.

Silva, B. (2015). Fusão cognitiva com impulso alimentar: Análise das propriedades psicométricas de uma nova medida e o seu impacto no comportamento alimentar (Dissertação de mestrado). Universidade de Coimbra, Portugal. Recuperado de https://estudogeral.sib.uc.pt/bitstream/10316/30996/1/ Disserta $\%$ C3\%A7\%C3\%A3o\%20B $\%$ C $3 \% \mathrm{~A} 1 \mathrm{r}$ bara $\% 20$ Leite $\% 20$ Silva.pdf

Tabachnick, B. G., \& Fidell, L. S. (2012). Using multivariate statistics. Person: New Jersey.

Timmerman, M. E., \& Lorenzo-Seva, U. (2011). Dimensionality Assessment of Ordered Polytomous Items with Parallel Analysis. Psychological Methods, 16, 209-220.
Tomás, I., Marsh, H. W., González-Romá, V., Valls, V., \& Nagengast, M. (2014). Testing measurement invariance across Spanish and English versions of the physical self-description questionnaire: An application of exploratory structural equation modeling. Journal of Sport \& Exercise Psychology, 36, 179-188. doi: 10.1123/jsep.2013-0070

Vandenberghe, L., \& Valadão, V. C. (2013). Acceptance, validation and mindfulness in contemporary cognitive behavior therapy. Revista Brasileira de Terapias Cognitivas, 9(2),126-135. doi: 10.5935/1808-5687.20130017

Wang, S., Chen, C. C., Dai, C. L., \& Richardson, G. B. (2017). A Call for, and Beginner's Guide to, Measurement Invariance Testing in Evolutionary Psychology. Evolutionary Psychological Science. (online). doi: 10.1007/s40806-017-0125-5

Recebido em: 02/05/2018

Reformulado em: 13/07/2018

Aprovado em: 29/08/2018 
Sobre os autores:

Evandro Morais Peixoto é docente do Departamento de Psicologia da Universidade de Pernambuco - UPE, coordenador do Laboratório de Avaliação Psicológica e Psicometria LAPPsi/UPE, doutor em Psicologia como Profissão e Ciência pela Pontifícia Universidade Católica de Campinas, com estágio doutoral PDSE desenvolvido na Université du Québec à Trois-Rivières - QC Canadá, mestre em Psicologia pela mesma universidade, com pós-doutorando em Psicologia pela Universidade São Francisco-USF.

ORCID: https://orcid.org/0000-0003-1007-3433

Email: peixotoem@gmail.com

Joel Gagnon é graduado em Psicologia pela Universté du Quebéc à Trois Rivieres UQTR - Quebec, Canadá, doutor em Psicologia, perfil pesquisador, pela mesma universidade. Desenvolve, ainda, trabalhos relacionados à psicométria e a análises estatísticas avançadas. Tem interesse de pesquisa nos temas Terapia de Aceitação e Compromisso (ACT) e procrastinação acadêmica.

ORCID: https://orcid.org/0000-0002-6961-0726

Email: gagnonjoel24@gmail.com

Tami R. Jeffcoat é psicóloga clínica e doutora pelo programa de Doutorado Reno em Psicologia Clínica pela University of Nevada UNR, Reno, Nevada, United States of America. Atualmente trabalha no desenvolvimento de tratamento, pesquisas e instrumentos de medidas em Terapia de Aceitação e Compromisso (ACT) e Terapia Comportamental Dialética (DBT).

ORCID: https://orcid.org/0000-0003-3385-9316

Email: doctorjeffcoat@gmail.com

Contato com os autores:

Evandro Morais Peixoto

Universidade de Pernambuco, Departamento de Psicologia

Av. Capitão Pedro Rodrigues, S/N, Magano

Garanhuns-PE, Brasil

CEP: 55294-902

Tel. (087) 3761-8229 